\title{
Arquitectura
}




\section{Hábitat / Teatro para la vida}

\section{Santiago Barrantes}

\section{Estudiante}

Escuela de Arquitectura. Universidad de Costa Rica (UCR)

Proyecto Académico del Taller de Diseño 9 (2015). Taller ARKHTEK. Profesores tutores: Arq. José A. Villarreal y Arq. Miguel Herrera

santialonso21@hotmail.com

Recibido: mayo del 2016

Aceptado: junio del 2016

\section{Arquitectura Proyecto Arquitectónico}




\section{Presentación}

El proyecto académico TEATRO PARA LA VIDA obtuvo una mención honorífica del Concurso de Diseño Arquitectónico (Exponer[NOS]) organizado por la Asociación de Estudiantes de Arquitectura (AEDA) de la Universidad de Costa Rica en mayo del 2016. Este proyecto fue realizado en el Taller de Diseño 9 (2015) de la opción ARKHTEK, esta opción parte de una metodología pedagógica, planteando el proceso de diseño y el desarrollo del proyecto arquitectónico en cuatro etapas: prediseño, investigación, diseño, reajuste y presentación. TEATRO PARA LA VIDA es una buena respuesta de ese proceso. Este proyecto está lleno de una alta sensibilidad en el tratamiento de sus espacios, en el uso de sus materiales y en una relación con el contexto de un profundo respeto.

Palabras Clave: arquitectura; contexto; espacios; materiales.

\section{Presentation}

The project "TEATRO PARA LA VIDA" won honorable mention in the Architectural Design Competition (Exponer[NOS]) organized by the Association of Students of Architecture (AEDA) of the University of Costa Rica in May of 2016. This project was designed in Level 9 Design Workshop organized by ARKHTEK, which utilizes a design process divided into four phases: predesign, research, design and presentation. "TEATRO PARA LA VIDA" is a good example of this process, with a highly sensitive treatment of space, use of materials and respectful relationship with its context.

Keywords: architecture; context; space; materials. 


\section{Hábitat / Teatro para la vida}

\section{Santiago Barrantes}

\section{Descripción del proyecto:}

Vivir el espacio, ser uno con él, emociones, sensaciones, alegría... Disfrutar de la vista, escuchar suavemente el agua caer y el viento que susurra entre los árboles, nada es quieto, todo está activo, el espacio es vida...

El agua me da frescura, el bambú y el viento me dan música, cuidar esa planta que crece y da vida a mi espacio, tocar la madera, sentir su textura y olor, observar como penetra la luz por esas pequeñas aberturas, siempre diferente, siempre impresionante...

El atardecer empieza a caer, puedo verlo entre el bambú, lo espero ansioso mientras el agua se va iluminando con su resplandor... Como el final de una obra de teatro, llega también el final de un día... Solo uno... Uno de muchos...
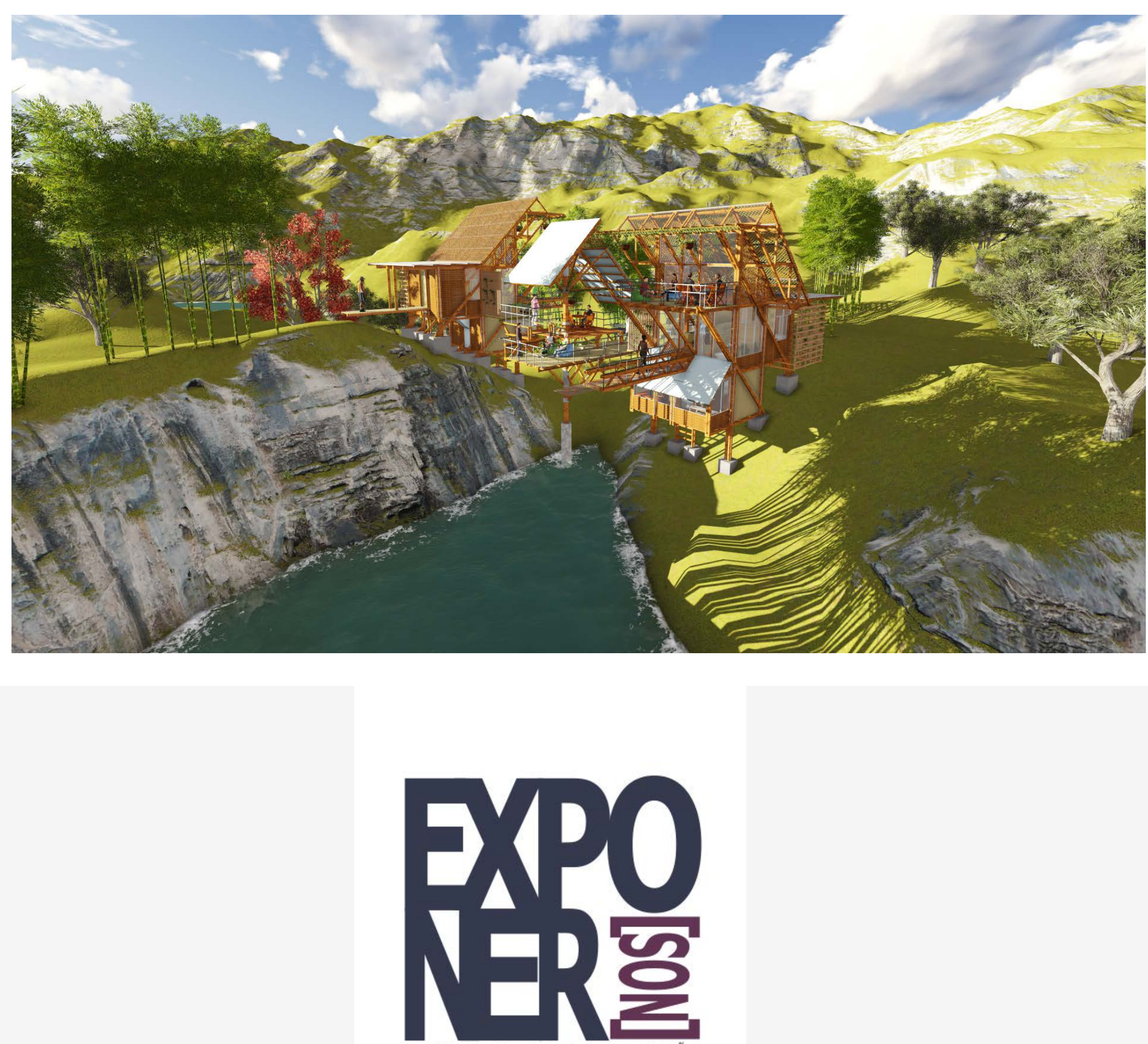

PRIMER CONCURSO DISEÑO ARQUITECTURA. UCR $\cdot 2016$ 

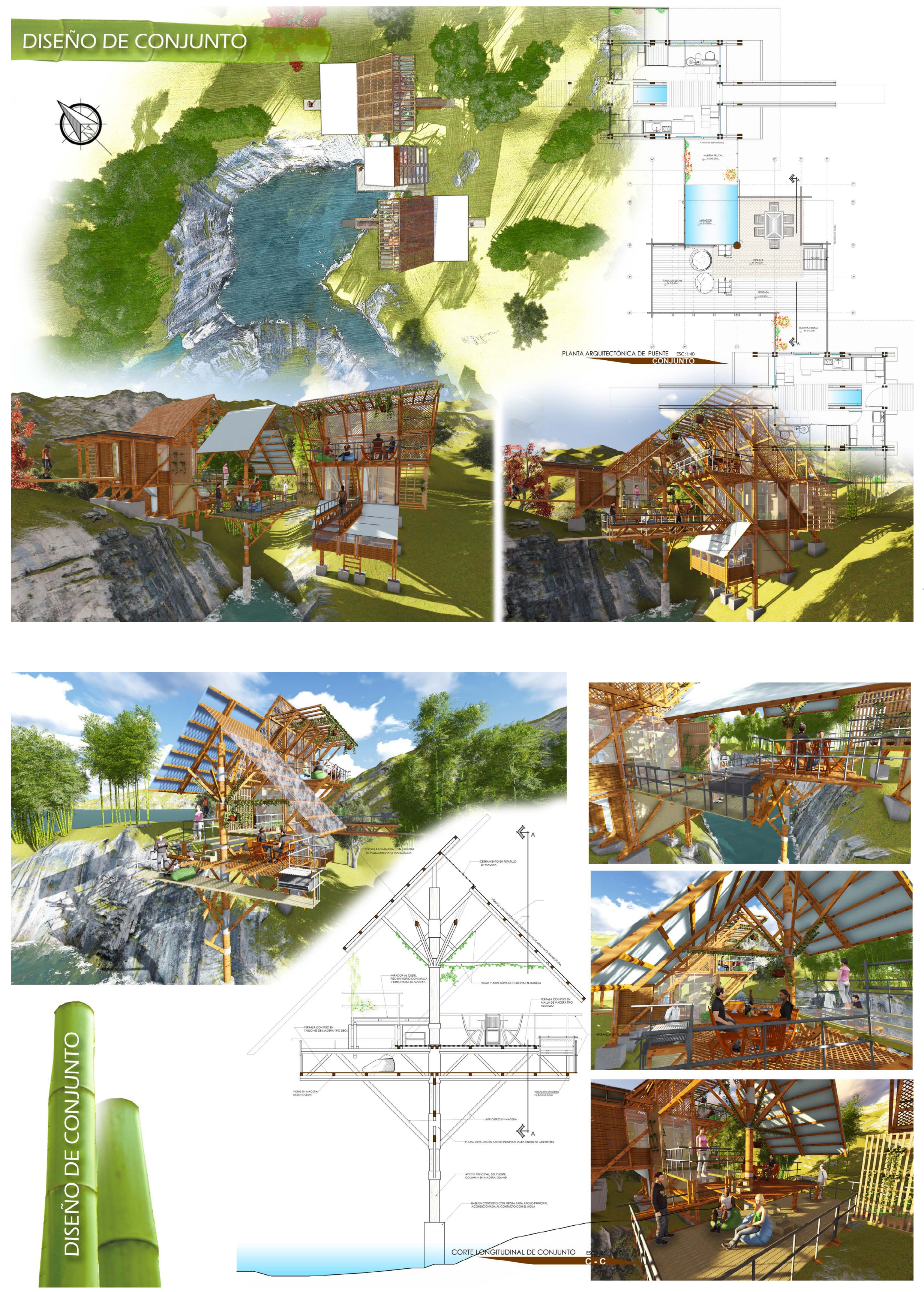
DISENO DE CONJUNTO Y MODULO
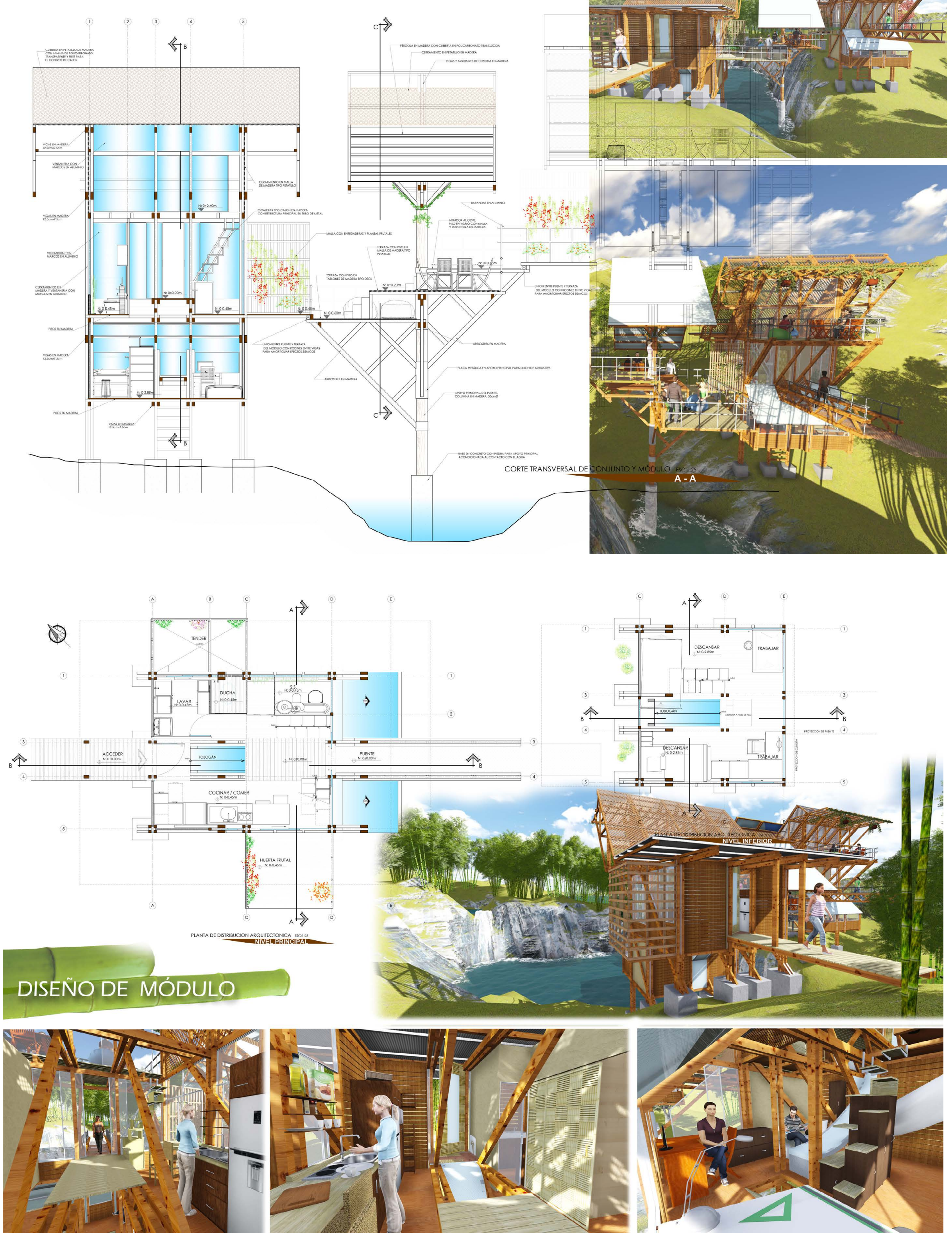

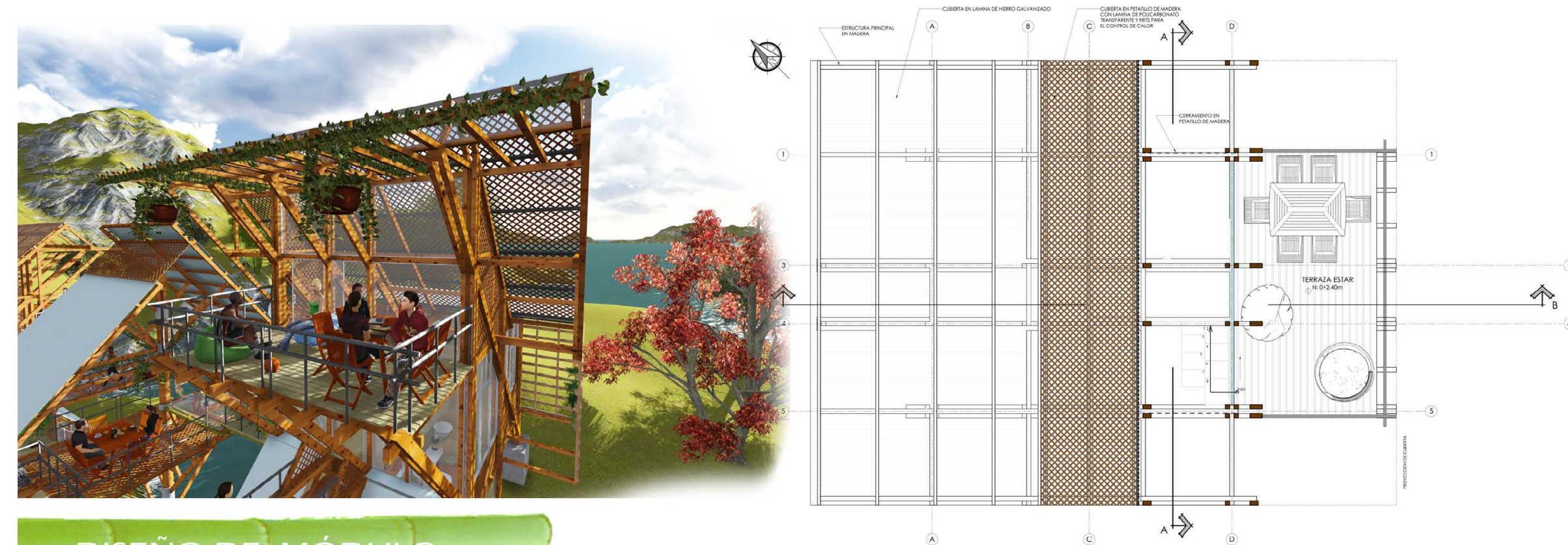

\section{DISEÑO DE MÓDULO}
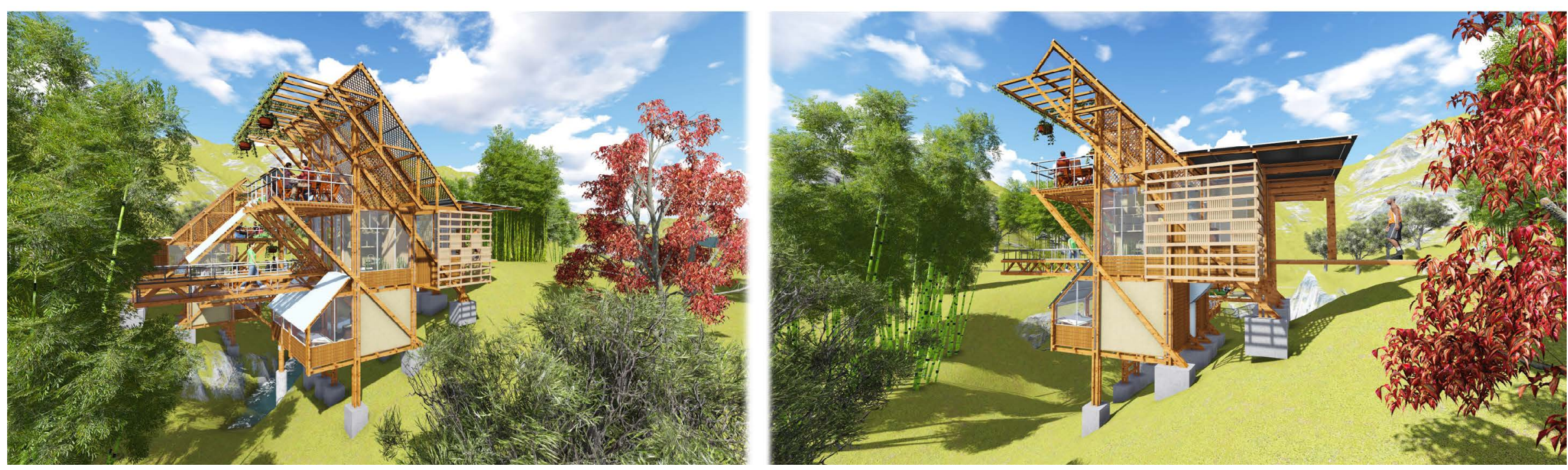

DISEÑODE MÓDULO
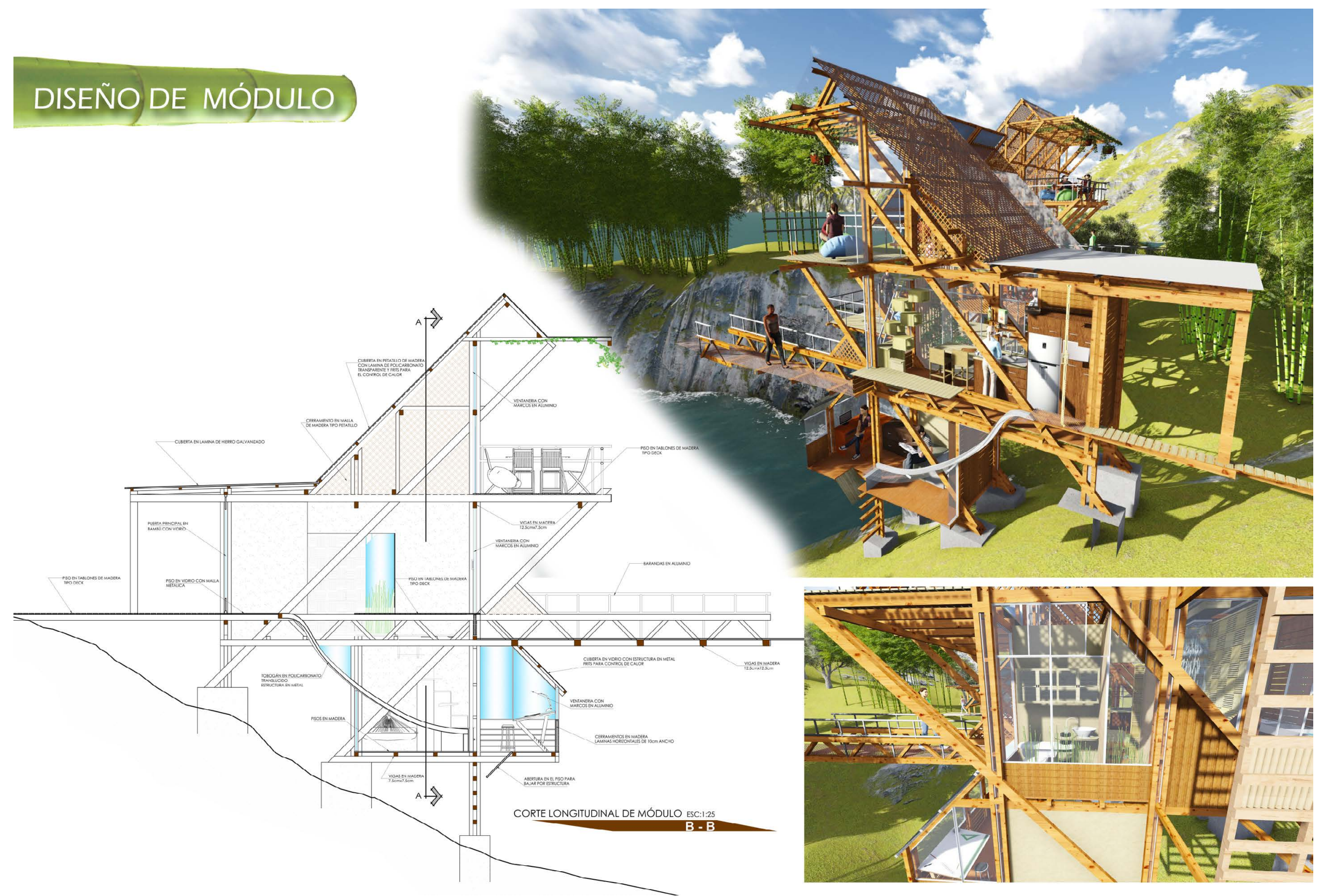
Este artículo forma parte de:

\section{REVISTARQUIS}

Para más información, artículos, e instructivo de publicación, visite:

www.arquis.ucr.ac.cr/revistarquis.html 